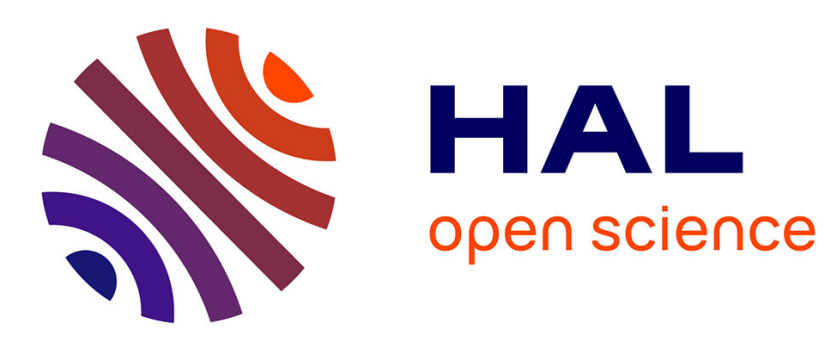

\title{
LE "PEAKING EFFECT"
}

\author{
C. Minier, J. Lauzier, C. Esnouf, Gilbert Fantozzi
}

\section{To cite this version:}

C. Minier, J. Lauzier, C. Esnouf, Gilbert Fantozzi. LE "PEAKING EFFECT". Journal de Physique Colloques, 1983, 44 (C9), pp.C9-51-C9-62. 10.1051/jphyscol:1983905 . jpa-00223327

\section{HAL Id: jpa-00223327 https://hal.science/jpa-00223327}

Submitted on 1 Jan 1983

HAL is a multi-disciplinary open access archive for the deposit and dissemination of scientific research documents, whether they are published or not. The documents may come from teaching and research institutions in France or abroad, or from public or private research centers.
L'archive ouverte pluridisciplinaire HAL, est destinée au dépôt et à la diffusion de documents scientifiques de niveau recherche, publiés ou non, émanant des établissements d'enseignement et de recherche français ou étrangers, des laboratoires publics ou privés. 


\title{
LE "PEAKING EFFECT"
}

\author{
C. Minier*o, J. Lauzier, C. Esnouf ${ }^{* *}$ et G. Fantozzi** \\ 'Centre d'Etudes Nuctéaires de Grenoble, Département de Recherohe Fondamentale, \\ Section de Physique du Solide, $85 X, 38041$ Grenoble Cedex, France \\ **Physique des Matémiaux, E.R.A. 463,I.N.S.A., Bâtiment 502, \\ 69622 Vizleurbanne Cedex, France
}

\begin{abstract}
Résumé - Les caractéristiques expérimentales du "Peaking Effect" sont passées en revue et les différents modèles théoriques d'interprétation sont analysés.
\end{abstract}

Abstract - The experimental features of peaking effect are reviewed and the theoretical models of interpretation are analyzed.

\section{I - INTRODUCTION}

Pendant longtemps, il a êté admis que 7 'influence des défauts créés par irradiation sur $i e$ fond de frottement interne $\left(Q^{-1}\right)$ se manifestait par une diminuation de $1^{\text {'amor- }}$ tissement due à 1 'ancrage des dislacations par les défauts. Cette image simple s'appuyait quantitativement sur des résultats de $Q^{-1}$ et module élastique (M) sous irradiation $Y$ d'un monocristal de cuivre entre 330 et $390 \mathrm{~K}$ et pour des fréquences de $11 \mathrm{kHz}(1)$. Au cours de 1 'irradiation on observait une variation linéaire du carré du défaut de module avec le frottement interne qui est bien prévue par le modèle théorique de Koehler (2) et Granato et Lücke (3) de mouvement des dislocations comme

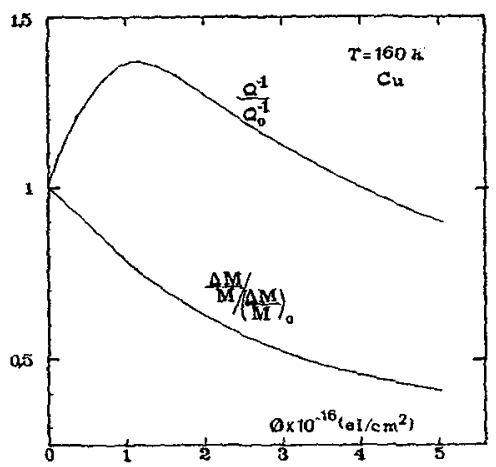

Fig. 1 - Défaut de module et FI normalisês, en fonction du flux d'électrons pour du cuivre écroui à $9 \mathrm{~K}(10)$. une corde vibrante $\left(10 i 1^{2}-1^{4}\right)$. Puis des déviations à la théorie sont apparues et la plus spectaculaire a été présentée par simpson et Sosin (4) : au cours d'un bombardement électronique de cuivre, le défaut de module montre bien la décroissance attendue alors que le frottement interne passe par un maximum $\left(Q_{M}^{-1}\right)$, ce phénomène a été baptisé "Peaking effect" $(P . E$.$) et un exemple est présenté sur la$ figure 1.

Depuis, un gros effort expérimental a été réalisé pour préciser les conditions d'existence et les caractéristiques de cet effet. L'essentiel est bien établi maintenant, bien que certains points soient controversés, nous alions développer ces résultats dans le chapitre II. Quant aux modèles théoriques qui tentent d'expliquer le phénomène, ils sont très nombreux et le domaine est encore très ouvert. Nous tenterons de faire le point sur cet aspect au cours du chapitre. III.

\section{II - CARACTERISTIQUES EXPERIMENTALES \\ 1 - Existence dans différents matériaux - Influence de l'état initial}

Le P.E. a tout d'abord été observé dans des métaux de structure c.f.c. et des

\footnotetext{
* Université Scientifique et Médicale de Grenoble
} 
échantillons polycristallins. Très net dans le cuivre (4), on peut l'observer dans l'aluminium, mais la décroissance correspondante du défaut de module y est beaucoup plus faible (5). Le P.E. est présent dans 7 'argent où il a été étudié en détaî (6), i) existe également dans le magnésium de structure hexagonale (7). Pour 7 'instant il n'a pas été observé dans les métaux cubiques centrés. Dans des monocristaux de cuivre le P.E. a été également observé (8).

L'effet des impuretés sur le P.E. n'a pas été étudié systématiquement mais il existe une comparaison entre l'argent $99,999 \%$ et $99,9999 \%$ qui montre bien que partant de niveaux initiaux $00^{-1}$ différents $l^{\prime}$ augmentation de frottement intérieur jusqu'au maximum du P.E. est sensibTement la même $(6)$.

L'influence de la structure métallurgique et en particulier de la température du recuit avant irradiation sur l'existence du P.E. a été initialement un point de désaccord entre les résultats expérimentaux de différents groupes. Pour l'un le P.E. $n$ 'existait pas dans le cuivre après recuit au-dessus de $750^{\circ} \mathrm{C}$ (5) alors que $7^{\prime}$ 'autre groupe l'observait après recuit à $900^{\circ} \mathrm{C}(9)$. La différence s'explique en fait par I'influence de la déformation de mesure : Te P.E. existe quelle que soit la température de recuit mais il apparaît pour des déformations qui dépendent de la température de recuit.

L'étude de 1'influence du taux d'écrouissage avant irradiation sur le P.E. a été faite dans le cuivre (10) irradié à $160 \mathrm{~K}$ sur des échantillons recuits ou diversement écrouis. Le frottement interne initial $0_{0}^{-1}$ est très vois in dans les différents cas et la conclusion est que I'amplitude du P.E. est d'autant plus faible que le taux d'écrouissage est plus important.

\section{2 - Influence de la déformation de mesure}

Dans les premières publications sur le cuivre, le P.E. a été présenté comme un phénomène indépendant de 7 'amplitude de mesure $\varepsilon$, puis en travaillant sur des échantillons recuits à plus haute température, dans lesquels l'effet de l'amplitude est plus important, i1 a été montré (9) que le P.E. n'apparaît que pour des valeurs de $\varepsilon$ supérieures à une certaine valeur critique et augmente ensuite avec $\varepsilon$ comme le montre la figure 2. Une certaine saturation de l'effet apparaît par exemple pendant $T^{\prime}$ irradiation à $115 \mathrm{~K}$. La saturation de l'amplitude du P.E. avec $\varepsilon$ a été confirmée par des expériences de Caro (11). Dans la fig. 2 , il faut noter que les valeurs initiales $Q_{0}^{-1}$ étaient indépendantes de $\varepsilon$ pour les irradiations à 160 et $115 \mathrm{~K}(9)$.

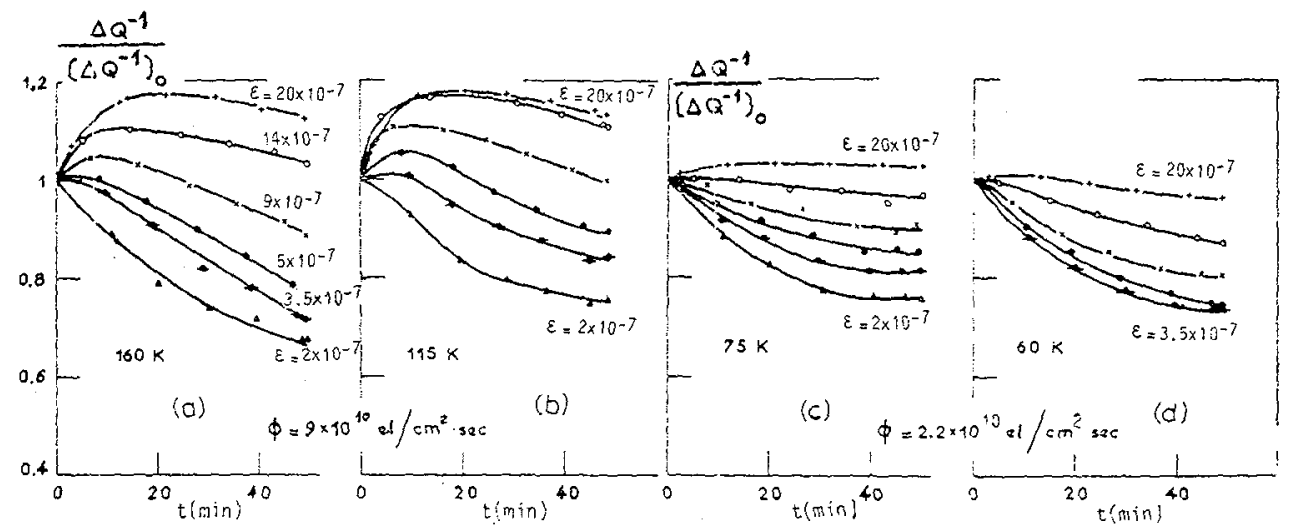

Fig. 2 - P.E. sur un échantilion de cuivre MRC recuit à $900^{\circ} \mathrm{C}$, pour différentes températures et déformations $\varepsilon(9)$.

Cette dernière remarque est en contradiction avec la conciusion d'expériences réaTisées dans le cuivre irradié à $350 \mathrm{~K}(11)(8)$ qui attribuent la dépendance du P.E. 
avec $\varepsilon$ à celle du fond initial $Q 0^{-1}$ avec $\varepsilon$. D'autres expériences reportées dans (10) montrent que pour plusieurs échantillons de cuivre irradiés à 160K, température à laquelle le frottement interne initial correspond au fond et où il ne dépend pratiquement pas de $E$, le P.E. dépend pourtant très fortement de $\varepsilon$ comme le montrent les figures 3 et 4 .

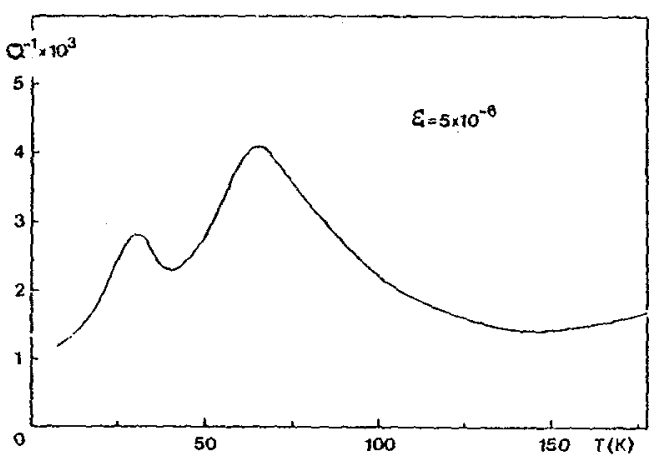

Fig. 3 - F.1. d'un échantillon de cuivre écroui $(10)$

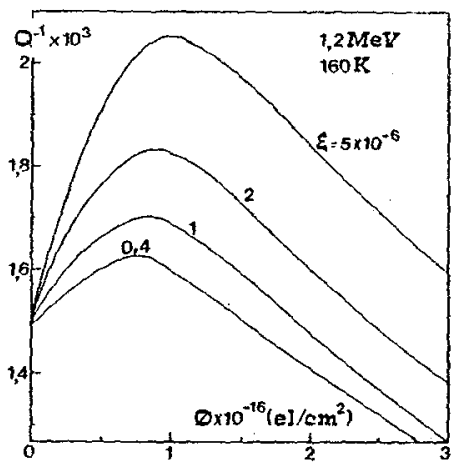

Fig. 4 - P.E. sur l'échantillon de la fig. 3 pour 4 déformations. FI non normal isé.

La conclusion expérimentale de ce paragraphe est nette : le P.E. dépend toujours de $\vec{E}$, il présente une saturation et il existe même dans des échantilions pour lesquels le fond de frottement interne initial ne dépend pas de $\varepsilon$.

\section{3 - Influence de la fréquence des mesures}

Les premières expériences semblaient indiquer que le P.E. était observable dans les expériences au $\mathrm{kHz}$ mais était absent au-delà. Une étude très détaillée de l'effet de la fréquence de mesure sur des échantillons de cuivre irradiés à $350 \mathrm{~K}$ a montré 7 'existence du P.E. depuis $1 \mathrm{kHz}$ jusqu'à $33 \mathrm{kHz}$ (12). L'amplitude du P.E. ne semble pas en rapport direct avec la fréquence mais parât liée à la valeur initiale $Q 0^{-1}$ avant irradiation : à une déformation $\varepsilon$ donnée, une relation empirique de type $Q_{m}^{-1} / Q_{0}^{-1}=1+A / Q 0^{-1}$ semble vérifiée.

\section{4 - Influence de Ia na ture des particules incidentes et de leur flux}

Le P.E. a été observé au cours de bombardement par des électrons d'énergie variable de 1 'ordre de 1 à $2 \mathrm{MeV}$ (4) (9) (11) ma is aussi pendant irradiation $Y$ (13).

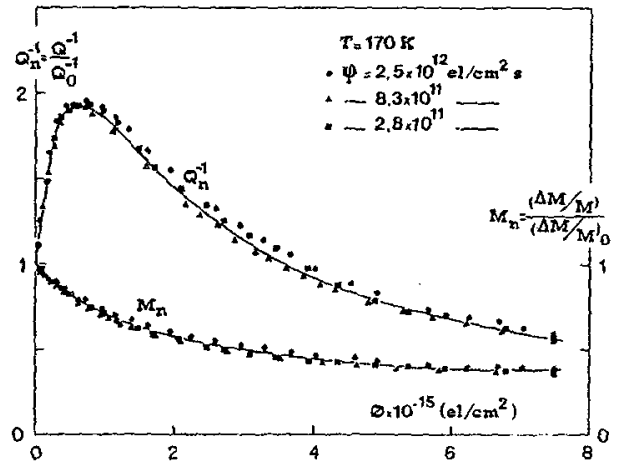

Fig. 5 - P.E. observé sur un échantilion d'argent au cours de 3 irradiations à des flux instantanés $\varphi$ différents (6).
L'influence du flux instantané $\varphi$ de particules incidentes a été étudié dans 1 'argent bombardé à $170 \mathrm{~K}(6)$. La fig. 5 montre que le paramètre important pour décrire le P.E. est le produit $\varphi t=\phi$, 7 'amplitude et la position du maximum restent les mêmes à différents flux instantanés; ce résultat a été confirmé dans d'autres conditions (11) en ajoutant qu'à certaines températures il pouvait y avoir une influence du temps $t$ seul sur lá Zème partie du P.E.

Le P.E. semble donc dû à la superposition de deux effets : la croissance du frottement interne attribué à un mécanisme de dissipation relié aux défauts présents au sein du cristal (ne dépend que du produit $\varphi \times t$ ) et 7 a décroissance liée à l'ancrage des dislocations par 
des défauts diffusant vers elles. Lorsque la température est telle que le temps de diffusion est de l'ordre de grandeur des durées d'irradiation, la dépendance avec le temps devient plus complexe.

\section{5 - Influence de la température d'irradiation}

Le P.E. existe pour tous les métaux étudiés sur une très large plage de températures. Dars le cas du cuivre, il a été observé jusqu'à $375^{\circ} \mathrm{C}$ (14). Lorsque la température décroît, il diminue en amplitude pour pratiquement disparaitre vers 60K (9). Par contre, dans certaines expériences qui ne sont pas faciles à reproduire, le P.E. a été reporté à basse température : $30 \mathrm{~K}(15)$ et $4 \mathrm{~K}(16)$ comme le montre la figure 6 . La variation thermique a été étudiée en détail dans 7 'argent bombardé aux électrons et la figure 7 montre également la disparition du P.E. vers $80 \mathrm{~K}$. Certains ont

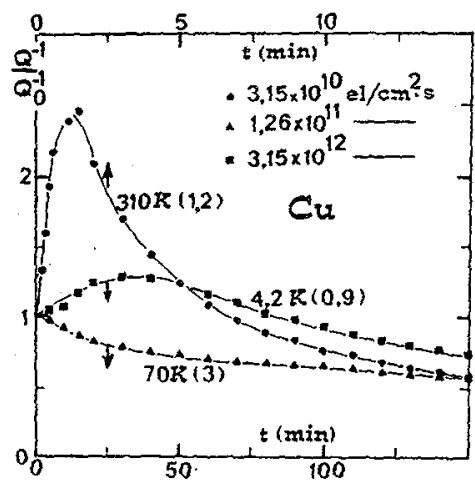

Fig. 6 - P.E. observé par Sosin à $4,2 \mathrm{~K}(16)$. Entre parenthèses : valeurs de $Q_{0}^{-1}$

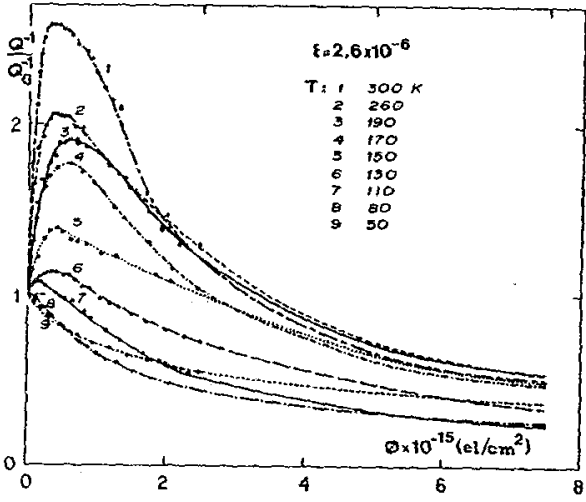

Fig. 7 - P.E. observé sur un échantillon d'argent à différentes températures (6).

relié la température de disparition du P.E. à la température du pic de Bordoni, d'autres à la température du stade IE de migration des interstitiels libres. Le tableau $\{$ montre les valeurs des températures correspondantes dans le cuivre, 1 'argent et le magnésium déduites de (9), (6) et (7).

Tab leau 1

TEMPERATURE DE DIFFERENTS PHENOMENES

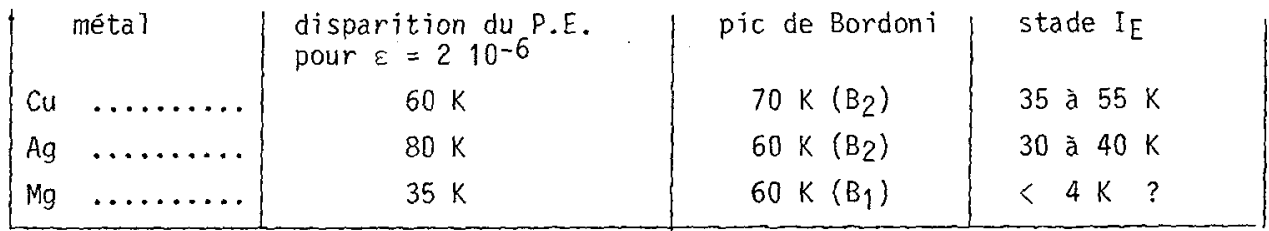

La corrélation ne semble pas pour l'instant assez nette pour trancher entre différents modèles mais il serait certainement très utile de faire des expériences complémentaires dans cette gamme de températures et à très forte déformation de mesure.

La variation entre la température du flux $\phi M$ correspondant au maximum du P.E. est qualitativement la même dans le cuivre et l'argent : $\phi M$ passe par un maximum pour une température de l'ordre de $160 \mathrm{~K}$ dans le cuivre (17) et $200 \mathrm{~K}$ dans 7 'argent (6). Ce maximum est associé à un minimum dans la vitesse d'ancrage (mesurée par Te module) en fonction de la température et peut ètre relié à une diffusivité de certains défauts ancreurs le long de certains types de dislocations bien déterminés (18). La réémission de certains défauts ancreurs vers le réseau doit être 
éventuel lement également prise en compte et de nombreux autres mécanismes peuvent encore compliquer l'analyse.

\section{6 - Relation entre le p.E__et_la création de_pics_de relaxation par irradiátion}

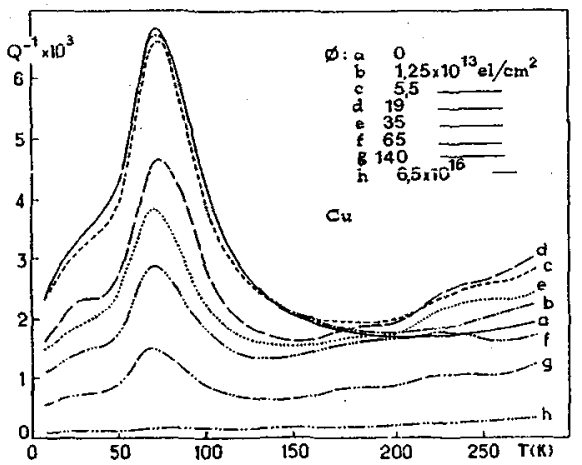

La large gamme thermique d'existence du $P . E$. exclut une relation directe du phénomène à un pic de frottement interne qui serait créé par irradiation comme le sont certains pics de type Hasiguti. Ceci a été mis clairement en évidence dans le cuivre bombardé par des électrons à 290K (10). Avant irradiation puis après chacune, je spectre de $\mathrm{Q}^{-1}$ a été tracé de 10 à 290K. La figure 8 montre la présence du P.E. à $290 \mathrm{~K}$ mais aussi qu'aucun pic de relaxation ne se construit et que Te P.E. correspond bien à une modification du fond de frottement interne.

Fig. 8 - FI en fonction de la température après différentes irradiations à $290 \mathrm{~K}$.

\section{7 - Les défauts responsables du P.E._sont-ils de nature interstitielelle

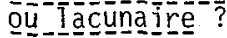

Ces deux types sont créés en nombre égal par T'irradiation, mais les interstitiels sont très mobiles à partir de $50 \mathrm{~K}$ environ dans $\mathrm{Cu}$ et $\mathrm{Ag}$ alors que les lacunes sont immobiles en-dessous de $200 \mathrm{~K}$ environ. Or, il n'y a pas de modification nette du P.E. vers $200 \mathrm{~K}$ et on est tenté de penser que les interstitiels jouent un rôle prépondérant. Ceci a été confirmé par des mesures de $Q^{-1}$ après trempe suivie ou non $d^{\prime} i r-$ radiation (19), les lacunes jouent essentiellement le rôle de points d'ancrage ferme sauf à forte déformation où des mécanismes de désancrage peuvent intervenir (20) et c'est aux interstitiels qu'il faudrait attribuer l'augmentation de $Q^{-1}$.

\section{III - MODELES D'INTERPRETATION DU PEAKING EFFECT}

De nombreux modèles ont été présentés depuis 1972, nous allons analyser ici leur capacité à expliquer toutes les caractéristiques expérimentales connues maintenant, i] ne faut donc pas oublier de les situer chronologiquement et c'est ainsi qu'ils vont être décrits.

\section{1 - Modèle_de_Simpson_et__ososin}

Le premier modèle du P.E. a été présenté en 1972 (4) en partant de la description du mouvement des dislocations par le modèle de la corde vibrante et l'équation

$$
A \frac{\partial^{2} y}{\partial t^{2}}+B \frac{\partial y}{\partial t}+K y=b \sigma_{0} e^{i \omega t}
$$

Leur hypothèse fondamentale était que les défauts créés par irradiation et arrivant sur les dislocations ne jouent pas le rôle de points d'ancrage supplémentaires, mais augmentent le frottement dans le mouvement de la dislocation. C'est à dire que la constante de rappel $K=\pi^{2} C / L^{2} n^{\prime}$ est pas modifiée, alors que le coefficient de viscosité $B$ augmente proportionnellement au nombre de défauts. Ce modèle explique 1 'existence du P.E. mais ne permet pas de décrire la dépendance avec $\varepsilon$. Par ailleurs Granato (21) a déduit les évolutions de $B$ et de $K$ au cours d'une irradiation à partir des variations de $Q^{-1}$ et de $\Delta M$ par les équations suivantes : 


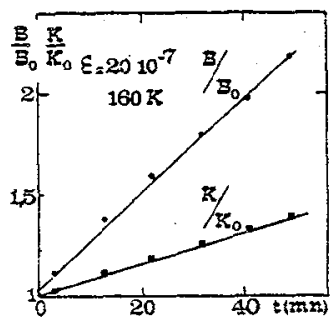

Fig. 9

$$
\frac{\omega B}{\Lambda M b^{2}}=\frac{Q^{-1}}{(\Delta M / M)^{2}+\left(Q^{-1}\right)^{2}} \frac{K}{\Lambda M^{2}}=\frac{\Delta M / M}{(\Delta M / M)^{2}+\left(Q^{-1}\right)^{2}}
$$

La figure 9 montre les variations de $B$ et $K$, au cours de l'irradiation d'un échantillon de cuivre a $160 \mathrm{~K}$. La variation du FI qui est donnée par la figure 2 présente un large "P.E.". On constate qu'au cours de l'irradiation on a une augmentation simultanée de $B$ et de $K$ qui est presque Tinéaire avec la dose de particules incidentes et on peut dire qu'au cours du P.E., I'arrivée des défauts s'accompagne de deux phénomènes, une diminution des longueurs libres des segments de dislocation et un effet de freinage supplémentaire.

\section{2 - Modèle de Seeger}

Dans ce modèle (22) le P.E. était relié à une amplification d'un effet Snoek de réorientation des auto-interstitiels se trouvant au voisinage d'une dislocation, par la contrainte périodique engendrée par le mouvement de la dislocation.

L'étude de la variation thermique du P.E. réalisée depuis, dans le cuivre et T'argent ne permet pas de conserver ce modèle, car le phénomène existe sur une gamme de température beaucoup trop grande pour un processus de ce type qui doit nécessairement être thermiquement activé.

\section{3 - Modèle de Mercier (23)}

Il considère que le mouvement des dislocations est décrit par celui des décrochements géométriques (D.G.) à basse température, alors qu'au dessus de la température du pic de Bordoni, interviennent à la fois les D.G. et les doubles décrochements (D.D.) créés. Ceux-ci n'apparaissent que si les longueurs libres des segments de dislocation satisfont la condition de Paré (24), c'est à dire si leur longueur L est supérieure à une 1ongueur critique $L_{c}$. Une dislocation de longueur $L$ engendrerait un $Q^{-1}$ plus important dans le modè le de déplacement de D.G. que par mouvement de D.D. L'irradiation diminuant $L$ certaines dislocations ont des longueurs qui deviennent inférieures à $L_{c}$ et leur mouvement passe d'un type D.D. à D.G. Dans certaines conditions et en particulier à température supérieure à celle du pic de Bordoni, ceci entraîne un "peaking effect".

Ce modèle a le mérite de permettre d'interpréter la variation de l'effet avec la déformation de mesure dans les échantillons bien recuits. En effet dans ce cas, les contraintes internes sont très faibles et c'est alors que leur rôle dans la condition de Paré est joué par la contra inte appliquée, ce qui explique la disparition du phénomène à faible déformation. Par contre on comprend moins bien l'effet de la contrainte de mesure qui est expérimentalement important pour un échantilion écroui, dans lequel les contraintes internes sont fortes. Enfin dans le cas du cuivre 1 'observation du P.E. à des températures inférieures à celles du pic de Bordoni permet d'écarter cette explication.

\section{4 - Modèle_de Frank_et_Gösele (25)}

La première idée de ce modèle est que des défauts lacunaires existent avant irradiation sur les dislocations coin et pas sur les dislocations vis qui interagissent beaucoup plus faiblement avec ces défauts. Les interstitiels créés par irradiation interaqissent avec les deux types de dislocations et 7 'hypothèse fondamentale retenue est qu'ils sont stables sur les dislocations vis et jouent donc le rôle de points d'ancrage fermes, alors que sous l'action de la contrainte mécanique, iTs migrent le long des dislocations coin et atteignent les défauts lacunaires. Donc au cours d'une irradiation les dislocations vis seraient ancrées, alors que les dislocations coins seraient désancrées. La troisième idée est que le frottement interne et le défaut de module sont la somme de contributions des dislocations vis et coin, mais qu'il est possible d'imaginer des conditions telles que $Q^{-1}$ reflète le mouvement des deux types de dislocations alors que $\Delta M$ serait essentiellement sensible 
à celuj des vis.

Dans ce modèle, la variation du P.E. avec $\varepsilon$ est expliquée par le fait que la diffusion des interstitiels le long des dislocations coin se fait sous 1 'influence de la contrainte mécanique. A très forte déformation les interstitiels diffusent et annihilent de façon irréversible les points d'ancrage lacunaires préexistants. A fàible déformation au contraire cette diffusion sera faible ou nulle et le P.E. n'apparaîtra pas.

Les expériences réalisées dans le cuivre montrant $1^{1}$ influence de la déformation par le P.E. et présentées sur la figure 2 ont été effectuées de la manière suivante : au cours d'une méme irradiation on mesure successivement le FI avec des déformations différentes. Dans ce cas, si Ta forte déformation provoquait un désancrage irréversible, la mesure suivante à faible déformation montrerait également ce phénomène donc une augmentation du frottement intérieur ce qui n'est pas observé.

Il n'est pas possible non plus dans ce modèle de rendre compte de l'indépendance du P.E. avec le flux instantané ni du très grand domaine thermique d'existence.

\section{5 - Modèle de Esnouf et Fantozzi}

Ce modèle phénoménologique repose sur 1 'existence de deux types d'interactions entre défauts ponctuels et dislocation (26). Certains défauts ponctuels (DP) diminuent le FI et les longueurs libres des dislocations par ancrage, d'autres localisés près du plan de glissement ralentissent les dislocations dans leur mouvement sans les ancrer. Ceci se traduit par un accroissement du temps de relaxation associé au mouvement des dislocations, qui est proportionnel au nombre de DP rencontrés, donc à l'aire balayée ff par les dislocations.

Le P.E. S'explique par une compétition entre le processus d'ancrage qui tend à réduire les effets de la relaxation, et le processus de "ralentissement" qui tend à accroitre l'énergie dissipée.

La description analytique de ce modèle se résume ainsi :

- L'ancrage des dislocations est décrit par la loi donnant la longueur moyenne L en fonction du nombre de défauts créés, c'est à dire du flux $\phi=\varphi \mathrm{t}$ : $L / L_{0}=1 /(1+a \varphi t)^{n}=f(\varphi t)=f(\phi)$ avec $a=$ Cte et $\varphi=$ flux instantané. Cette roi est connue par 1 'expérience : $\Delta / \Delta_{0}=K_{0} / K=\left(L / L_{0}\right)^{2}=f^{2}(\phi)(\Delta=$ intensité de relaxation et $K$ terme de rappel dans le modèle de la corde) et au cours d'une irradiation 7 'évolution de $K_{0} / K$ est donnée par la relation 1 .

- L'accroissement du temps de relaxation est $\Delta \tau=C$ avec $C \propto p=\rho_{0} t^{m}$, $\rho$ étant la densité de défauts freineurs produits par irradiation :

Ainsi : $\Delta \tau \propto \frac{L}{h b} \varepsilon_{d} t^{m}$ et $\varepsilon_{d}=\Delta \frac{\varepsilon}{\sqrt{1+\omega^{2} \tau^{2}}}$

$\left(i=\right.$ densité de dislocations $; \varepsilon_{d}=$ déformation instantanée due aux dislocations et $\varepsilon=$ déformation de mesure).

soit $\Delta \tau \propto \frac{L^{3} \varepsilon t^{m}}{\sqrt{1+\omega^{2} \tau^{2}}}=k_{0} \frac{\varepsilon t^{m} f^{3}(\phi)}{\sqrt{1+\omega^{2} \tau^{2}}}$

avec $k_{0} \propto L_{0}^{3}=$ Cte

L'anomalie de module et le F.I. sont alors décrits par les relations classiques avec le temps de relaxation donné par

$$
\tau=\tau_{0}+\frac{B_{0}}{R}+\Delta \tau=\tau_{0}+\frac{B_{0}}{K_{0}} f^{2}(\varphi t)+k_{0} \frac{\varepsilon t^{m} f^{3}(\varphi t)}{\sqrt{1+\omega^{2} \tau^{2}}}
$$

avec $\tau_{0}=$ temps de relaxation associé à un mécanisme (Bordoni par exemple)

$B_{0}=$ terme de frottement visqueux à 7 'origine du F.I. initial. 
On remarque qu'aux températures très supérieures à celle du maximum du pic de relaxation $\left(\omega \tau_{0}<<1\right) ~ I ' e x i s t e n c e$ possible du $P$. E. est liee au 3ème terme de $\tau$ alors que le 2ème (puis le 3ème) sont responsables de la chute du F.I. pour des temps plus longs.

Un exemple de P.E. est donné par la figure 10 lorsque $Q_{0}^{-1} \simeq \Delta_{0} \omega \mathrm{B}_{0} / K_{0}\left(\tau_{0} \simeq 0\right)$. On remarque 1 'importance du paramètre

$$
x=\omega k_{0} \varepsilon \Delta_{0} / Q_{0}^{-1}=k_{0} \varepsilon K_{0} / B_{0}
$$

Toutefois, afin de préciser 1 'importance des paramètres $\varepsilon, Q_{0}^{-1}$ et $L_{0}$ il est intéressant de calculer une expression approchée de $Q^{-1} / Q_{0}^{-1}$. Lorsque le F.I. reste fai-

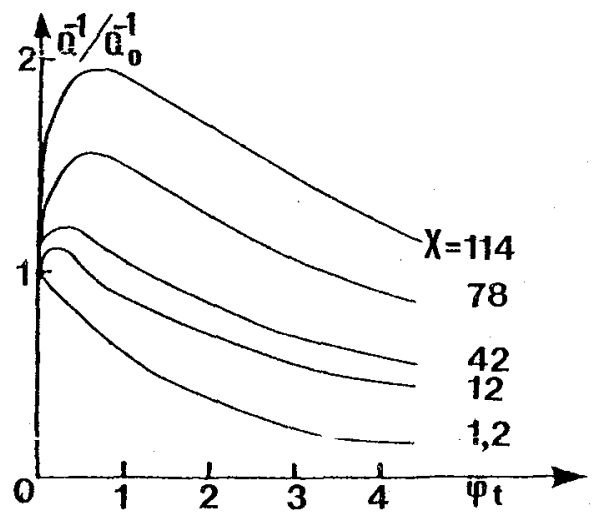

Fig. 10 - Courbes théoriques du P.E. dans le cas : $a=710^{-16} \mathrm{~cm}^{2}$; $n=0,3 ; m=2 / 3 ; Q_{0}^{-1}=10^{-3} \Lambda^{\prime 2}$. ble $(\omega t<<1)$ on peut écrire:

$\frac{\Delta M / M}{(\Delta M / M)_{0}}=\frac{\Delta}{\Delta_{0}}$ soit $\frac{\Delta M / M}{(\Delta M / M)_{0}^{-}} \simeq f^{2}(\varphi t)$

$\frac{Q^{-1}}{Q_{0}^{-1}} \simeq \frac{\Delta}{\Delta_{0}} \frac{\tau}{B_{0} / K_{0}}$ ou $\frac{Q^{-1}}{Q_{0}^{-1}}=f^{4}\left(\varphi_{t}\right)\left[1+x t^{m} f\left(\varphi_{t}\right)\right]$

Ainsi, hormis m et $f(\varphi t)$ (irradiation) seu? $x$ intervient, donc les grandeurs $\varepsilon$, $k_{0} \propto L_{0}^{3}$ et $1 / Q_{0}^{-1}$ ont les mêmes influences sur le P.E. En particulier si $Q_{0}^{-1}$ dépend de $\varepsilon$, le P.E. en dépendra aussi, mais cette dépendance n'est pas nécessaire pour l'observer. L'indépendance du P.E. vis à vis de la fréquence demande que $K_{O} / B_{O}$ (ou $\omega \Delta_{0} / Q_{0}^{-1}$ ) reste constant (relation 4 ). Pour préciser ce point, il serait souhaitable de mesurer le F.I. en fonction de la fréquence mais aussi la variation de l'intensité de relaxation $\Delta_{0}$.

\section{6 - Modèle_de_Caro et Mondino}

Ce modèle (11) (12) suppose que 7 'énergie dissipée au cours d'un cycle du mouvement d'une dislocation de longueur $L_{0}$ est proportionnel au nombre de 1 iaisons atomiques dans le plan de glissement qui sont cassées quand la dislocation les balaie : $W=2 a_{0} / b^{2}$, étant 1 'aire balayée et $a_{0} 1$ 'énergie irradiée par liaison cassée. Ceci entraine un fond de F.I. $Q_{0}^{-1}$ et un défaut de module $\triangle G / G$ de la forme :

$$
Q_{0}^{-1}=\frac{2 a_{0} \Lambda L_{0}^{2}}{3 G b^{3} \varepsilon} \quad \frac{\Delta G}{G}=\frac{1}{6} \alpha \Lambda L_{0}^{2}
$$

Durant une irradiation il y a, comme dans le modèle de Esnouf, une double action des défauts créés, ceux dans le réseau $n_{L}(t)$ et ceux qui ancrent les dislocations $n_{D}(t)$. Ils provoquent une modification de $a_{0}$ et $L_{0}$ qui deviennent $a_{0}^{\prime}=a_{0}+a_{1} n_{L}(t)$ et $L=L_{0} / 1+n_{0}(t)$.

En outre, il y a un effet de $\varepsilon$ sur Lo et $n_{0}(t)$ traduisant le désancrage des ancreurs initiaux et de ceux dus à 1 'irradiation. Ceci entraine : $L_{0}(t=0, \varepsilon)=\left(L_{00}\left(\varepsilon / \varepsilon_{n}\right)^{N}\right.$ et $L_{o}(t, \varepsilon)=\frac{L_{0}(t=0, \varepsilon)}{1+n_{D}(t)\left(\varepsilon / \varepsilon_{n}^{\prime}\right)-M}$ ( $\varepsilon_{n}$ et $\varepsilon^{\prime} n$ sont des facteurs de normalisation); ce qui conduit à un FI de la forme :

$Q^{-1}(t, \varepsilon)=Q_{00}^{-1}\left(\frac{\varepsilon}{\varepsilon_{n}}\right)^{2 N-1} \frac{1+A t}{\left[1+B\left(\varepsilon / \varepsilon_{n}\right)^{N-M} t\right]^{2}}$ 
avec $A$ tel que $n_{L}(t)=A\left(a_{0} / a_{1}\right) t ; B=d L_{0 o} / \Lambda ; Q_{00}^{-1}$ est le fond de $F I$ à $t=0$ et $\varepsilon=\varepsilon_{n}$. L'exposant $N$ est obtennu à partir de la dépendance avec $\varepsilon$ du FI initial $Q_{0}^{-1}=Q^{-1}(t=0, \varepsilon)=Q_{0}^{-1}\left(\varepsilon / \varepsilon_{n}\right)^{2 N-1}$ et $M$ à partir de $1^{\prime}$ 'état irradié $Q^{-1}(t=\infty, \varepsilon)$.

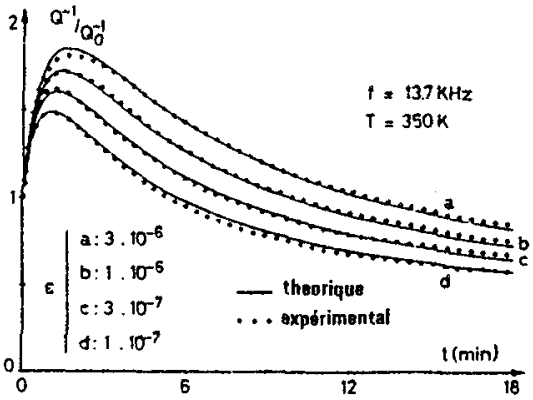

Fig. 11 - P.E. calculé par Caro et al. (11) (13) (pour des irradiations à 4 déformations différentes sur du cuivre polycristallin) avec les valeurs : $N=0,52 ; M=0,6 ; A=3,22 \mathrm{~min}^{-1}$ et $B=0,48$ min $^{-1}$.
La figure 11 donne un exemple de P.E. obtenu à partir de la relation 7 .Cel le-ci montre clairement que les variations du P.E. et celles du fond de FI $Q_{0}^{-1}(\varepsilon)$ correspondent au même phénomène. L'augmentation de 1 'amplitude du P.E. avec $\varepsilon$ ne peut s'expliquer que par celle de $0_{0}^{-1}$ : si celui-ci est constant (alors $N=1 / 2$ ) 1 'amplitude du P.E. est indépendante de $\varepsilon$ (8). En outre, Te P.E. apparait quand $M>N, M+N>1$ (en général $M>1 / 2$ et $N>1 / 2$ ), ces conditions sont donc très critiques. Le modèle de Caro et Mondino rend compte de l'évolution du P.E. avec la tempéra-

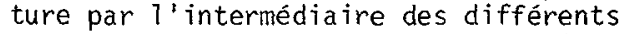
mécanismes de diffusion des DP et d'ancrage de dislocations. Enfin le P.E. décrit par ce modèle ne dépend pas de la fréquence puisque l'énergie dissipée choisie n'en dépend pas non plus.

\section{7 - Modèle de Ritchíe}

Ritchie et al (27) ont développé un modèle de mouvement zig-zag d'une dislocation dans un arrangement d'atomes de soluté. La dislocation saute d'un obstacle attractif à un autre obstacle attractif par dessus un obstacle répulsif. La vitesse de déplacement de la dislocation est donnée par :

$$
\dot{y}=Z w o \sinh \left(b^{3} \sigma_{e f f} / c k T\right)
$$

avec $Z=$ amplitude du mouvement $z i g-z a g, \omega_{0}=$ fréquence de saut à $\sigma=0, b=$ vecteur de Burgers, $c=$ concentration d'obstacles et $\sigma_{\text {eff }}=$ contrainte effectivel=contrainte appliquée moins contrainte de rappel).

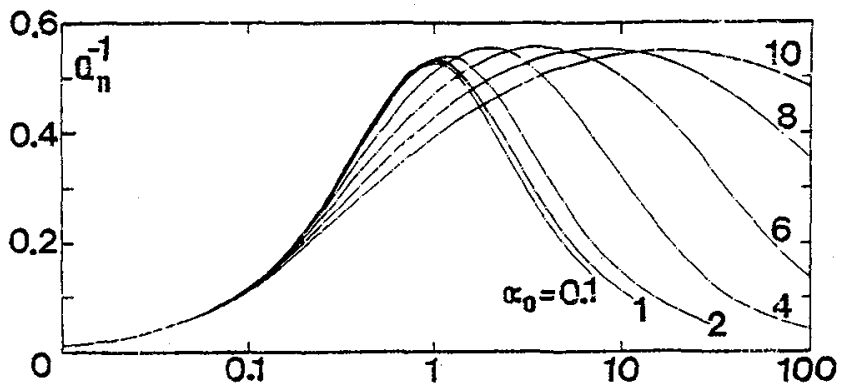

Fig. 12 - FI normalisé en fonction de $\omega \tau$ pour différentes valeurs de $\alpha_{0}=\sigma_{0} b^{3} / \mathrm{ckT}$.

Quand $\varepsilon>c k T / E b^{3}$, la mobilité des dislocations dépend de $\varepsilon$ et l'équattion n'est plus linéaire. La figure 12 donne le FI calculé par Ritchie et a1 (27).

Ils obtiennent un pic de FI présentant des effets non linéaires importants dont la position dépend de la concentration en atomes de soluté. Quand $\varepsilon$ devient faible, on tend vers un pic de Debye qui correspond à 1 'interaction des dislocations uniquement avec les obstacles attractifs. Le modèle conduit à un P.E. situé sur le côté basse température du pic ( $\omega \tau$ grand), qui n'apparaît qu'à partir d'une certaine contrainte. Ainsi, le P.E. n'existe que dans un domaine restreint de température car il s'agit d'un phénomène thermiquement activé $\left(\tau \propto \tau_{0}\right.$ exp $U / k T, U$ étant 1 'enthalpie effective d'activation). Ce modèle ne permet donc pas d'expliquer les résultats expérimentaux. 


\section{8 - Modèle_de_Zil berman et_L_Levin}

Ces auteurs (28) ont développé une théorie de 7 'amortissement dû aux dislocations indépendant de 1 'amplitude de solTicitation en tenant compte d'une atmosphère de $D . P$. autour de la ligne. Les D.P. sont classés en deux groupes : les défauts ancrant les dislocations (force d'ancrage très supérieure aux autres termes de 7 'équation) et les défauts plus loin du coeur de la dislocation avec laquelle ils interagissent.

En linéarisant la force d'intéraction l'équation du mouvement s'écrit :

$$
\left[B \frac{\partial}{\partial t}-T_{1} \frac{\partial^{2}}{\partial x^{2}}+V(x)\right] y(x, t)=\sigma b \text {. }
$$

où $B$ est le terme d'amortissement indépendant de $l^{\prime}$ 'atmosphère de $D P$, $T_{1}$ la tension de ligne et $y(x, t)$ fixe la forme de la ligne de dislocation.

Aux basses fréquences : $\omega \ll \omega_{1}=\left(A^{2} / 8 T_{1} B^{3}\right)^{1 / 3}$ (où $A \propto c W^{2}: c$ = concentration de D.P, $W_{0}=$ énergie de liaison), le coefficient de frottement visqueux effect if $B^{*}$ est essentiellement déterminé par l'énergie dissipée lors de l'intéraction avec le champ élastique des D.P., et $B^{*} \propto B$ W / $/ \omega$ et dépend donc de $c$ la concentration de D.P., ce qui conduit à un P.E. (comme dans la théorie de Simpson et Sosin) mais qui est indépendant de la fréquence $\left(B^{\star} \propto 1 / \omega\right)$. Par contre, ce modèle n'explique pas le comportement du P.E. vis à vis de la contrainte de sollicitation.

\section{IV - DISCUSSION}

Les caractéristiques du P.E. peuvent être présentées ainsi :

\section{A - Carractéristigues principales}

1 - Le P.E. est directement lié au nombre de défauts introduits, (dans le cas d'une irradiation 11 est fonction de $t=\phi=$ flux intégré).

2 - Son amplitude croit généralement avec la sollicitation $\varepsilon$ (il existe une contrainte critique nécessaire à son apparition).

3 - Il existe dans un très grand domaine de températures, généralement il commence à apparaître entre 40 et $80 \mathrm{~K}$ suivant les métaux étudiés (cf tableau I) et son amplitude dépend de la température.

quences.

4 - Il existe avec une amplitude comparable dans un grand domaine de fré-

\section{B - Caractéristigues secondaires :}

1 - La contrainte critique d'apparition et 1 'amplitude du P.E. sont liées à l'état microstructural initial.

2 - Bien qu'il n'existe généralement qu'à partir d'une certaine température, 11 a quelquefois été observé à basse température (4K).

mations.

3 - Il existe une saturation de l'amplitude du P.E. pour les fortes défor-

On peut classer les modèles de la manière suivante :

- ceux attribuant le P.E. à un mouvement thermiquement activé des dislocations et/ou des défauts ponctuels (Seeger, Ritchie, Frank);

- ceux qui sont basés sur un mode de frottement athermique (Sosin, Mercier, Esnouf, Caro et Zilberman).

Le premier groupe de modèles ne peut être retenu en vertu des caractéristiques $A_{3}$ et 
$A_{4}$ (quelquefois $A_{2}$ ). Dans le cadre de ces modèles le domaine de température où existe le phénomène est beaucoup trop étroit (restauration des défauts nécessaires ou activation thermique).

Dans le second groupe, nous devons distinguer les modèles linéaires (ceux de Mercier, de Simpson Sosin repris par Ogurtani (29) et de Zilberman et Levin) qui ne peuvent convenir à cause de $A_{2}$ et aussi $A_{4}$ pour ceux de Mercier et de Sosin. Les 2 derniers modèles (Esnouf et Caro) admettent que l'énergie dissipée par cycle est corrélée au nombre de D.P. rencontrés par la dislocation, les rendant ainsi non linéa ires. Nous allons les discuter plus en détail.

Le modèle de Caro et Mondino explique très bien l'existence du P.E. (avec une amplitude comparable) dans un grand domaine de fréquences.

Par contre, ces auteurs ont montré (8) que la variation de l'amplitude du P.E. $\left(Q \bar{M}^{-1} / Q_{0}^{-1}\right)$ avec $\varepsilon$ ne dépend que de celle de $Q_{0}^{-1}$, or 1 'expérience fig. 2 et 3 montre que 1 'on peut avoir un P.E. fortement dépendant de la déformation aiors que $Q_{0}^{-1}$ en est indépendant. En outre, leur modèle n'explique pas la saturation possible du P.E. avec $\varepsilon$.

Ce modèlè rend compte de 1 'évolution du P.E. avec la température par l'intermédiaire des cinétiques de diffusion des défauts. Les calculs de Caro (30) montrent un bon accord avec l'expérience dans le domaine de température autour du stade III, cependant ce modèle $n$ 'explique pas correctement les conditions d'apparition du P.E. avec la température (31).

Enfin à déformation constante, le fond initial de FI est proportionnel à $L_{0}^{2}$ comme le défaut de module, or expérimentalement pour les très faibles déformations, il a été montré (9) que le FI variait comme le carré du défaut de module.

Quant au modèle de Esnouf et Fantozzi, il donne très bien la dépendance du P.E. avec $\varepsilon$ : position et amplitude du maximum, absence du P.E. pour les faibles déformations. En particulier une dépendance de $Q_{0}^{-1}$ avec $\varepsilon$ entraîne une variation du P.E. ma is cette dépendance n'est pas nécessaire pour observer une variation du P.E. avec $\varepsilon$. Enfin il explique la saturation possible de l'amplitude du P.E. en fonction de $\varepsilon$ qui a été observée expérimentalement.

Ce modèle décrit correctement la dépendance du P.E. avec la température : apparition du P.E. à une température voisine de celle d'un pic de Bordoni, existence possible du P.E. à basse température liée à la relaxation des décrochements géométriques.

Il reste à vérifier si ce modèle peut rendre correctement compte de l'existence du P.E. dans un très grand domaine de fréquence (quelques centaines de $H_{z}$ à quelques dizaines de $\mathrm{kHz}$ ) il est souhaitable de mesurer le $\mathrm{FI}$ initia? $\mathrm{Q}_{0}^{-1}$ ma is aussi l'intensité de la relaxation $\Delta_{0}$ en fonction de la fréquence de manière à connaitre l'évolution du paramètre $x=\omega k_{0} \varepsilon \Delta_{0} / 0_{0}^{-1}$ (rel. 4 ).

Bien que le modèle de Zilberman soit linéaire, il donne une interprétation possible de la dissipation d'énergie responsable du P.E. et conduit à un effet indépendant de la fréquence. Un développement plus complet dans le domaine dépendant de 1 'amplitude serait très intéressant.

En conclusion, les modèles susceptibles d'expliquer les caractéristiques du P.E. sont phénoménologiques et font intervenir deux types de défauts, l'un ancrant les dislocations et 1 'autre dissipant de 7 'énergie. Cependant, des efforts expérimentaux et théoriques sont encore nécessaires pour déterminer les mécanismes physiques exacts et la nature des défauts qui interagissent avec les dislocations. 


\section{REFERENCES}

( 1) THOMPSON D.O., BUCK O., BARNES R.S. et HUNTINGTON R.B., J. App . PhyS. 38 , (1967) 3051 .

( 2) KOEHLER J.S., Imperfections in Nearly Perfect Crystals (New York : Wiley) (1952) p. 197.

( 3) GRANATO A.V. and LÜCKE K., J. Appl. Phys. 27 (1965) 583.

(4) SIMPSON H.M., SOSIN A. et JOHNSON D.F., Phys. Rev., B $\underline{5}$ (1972) 1382 et 1393.

( 5) SEIFFERT S.L., SIMPSON H.M. et SOSIN A. J. Appl. Phys. 44, (1973) 3404.

( 6) GIRARD P. et MINIER C., Journ. de Phys. 39 (1978) 981.

( 7) MINIER C., LAUZIER J., ESNOUF C. et FANTOZZI G., Phys. Stat. Sol. (a) 71 (1982) 381.

( 8) CARO J.A., GLASS N.E. et MONDINO M.A., J. App1. Phys. 53, (1982) 4854.

( 9) LAUZIER J., MINIER C. et SEIFFERT S.L., Phil. Mag. 31, (1975), 893.

(10) LAUZIER J., Thèse Université de Grenoble (1981).

(11) CARO J.A. et MONDINO M., J. App1. Phys. 52, (1981) 7147.

(12) CARO J.A., Thèse Ecole Polytechnique de Lausanne (1981).

(13) CARO A. et MONDINO M., Scripta Met. 13 (1979) 1073.

(14) SIMPSON H.M. et KERKHOFF S.J., Rad. Effects 27 (1976) 191.

(15) MINIER C., II Nuovo Cimento 33 B,1 (1976) 109.

(16) SOSIN A. et SIMPSON H.M., J. Appl. Phys. 49, 1 (1978) 188.

(17) SIMPSON H.M., SOSIN A., SEIFFERT S.L., J. Appl. Phys. 42 (1971) 3977.

(18) LAUZIER J., GIRARD P. et MINIER C., J. de Physique 10, 42 (1981) C5-247.

(19) SIMPSON H.M. et KERKHOFF S.J., Phys. Rev. Lett. 33, (1974) 155.

(20) KERANS R.J. et SIMPSON H.M., J. Appl. Phys. 50 (1979) 4739.

(21) GRANATO A.V., Fundamental Aspects of Radiation Damage in Metals, ed. Robinson M.T. et Young F.W. ERDA report Conf. 751006 , Gat1inburg 11 (1975)932.

(22) SEEGER A., Internal Friction and Ultrasonic Attenuation in Solids ed : Lenz D. et Lucke K. Springer-Verlag. Berl in, 2, (1975) 232.

(23) MERCIER 0., Phi1. Mag. A, 37 (1978) 813.

(24) PARE V.K., J. AppT. Phys., 32 (1961) 332.

(25) FRANK $W$. et GÖSELE U., Internal Friction and UTtrasonic Attenuation in Solids, et : C.C. Smith, Pergamon Press Manchester (1980) 109.

(26) ESNOUF C. and FANTOZZI G., Internal Friction and U1trasonic Attenuation in Solids, ed C.C. Smith, Pergamon Press, Manchester (1980) 103.

(27) RITCHIE I.G., ATRENS A., SO C.B. and SPRUNGMANN K.W., Internal Friction and Ultrasonic Attenuation in Solids, J. Phys. C5, 42 (1981) 319.

(28) ZILberman L.A. and LeVin L.G., J. Phys. F : Met. Phys. 13 (1983) 1127.

(29) OGURTANI T. 0 ., Phys. Rev. B 21 (1980) 4373.

(30) CARO J.A., J. Phys. F : Met. Phys. 13 (1983) 1115.

(31) ESNOUF C. et FANTOZZI G., ECIFUAS 4, J. Phys. (cette conférence). 